\title{
Vasoactive intestinal peptide in canine portosystemic shunt in the absence of portal hypertension
}

\author{
Mitsuhiro Isaka* and Hiroshi Ueno \\ Department of Small Animal Clinical Sciences, School of Veterinary Medicine, Rakuno Gakuen University, \\ Ebetsu, Japan
}

\begin{abstract}
Background: The congenital portosystemic shunt (PSS) is a common vascular anomaly in dogs. Vasoactive intestinal peptide (VIP) is produced in various organs (including the small intestine, large intestine, and pancreas), leading to abdominal vasodilation, increased blood flow, increased pancreatic blood flow, and promotion of pancreatic endocrine and exocrine secretions. However, there have been no reports on the concentration of VIP in the portal and peripheral veins in canine PSS.

Aim: The aim of this pilot study was to evaluate whether dogs with PSS have a different VIP concentration in their portal system in general.

Methods: Six dogs with an extrahepatic portosplenic shunt were included in the study. Blood samples were taken from the saphenous and portal veins during PSS ligation surgery with an amerid constrictor, to evaluate and compare the VIP concentration in both samples. VIP was measured using a commercial canine enzyme-linked immunosorbent assay kit. Results: The breeds included Mongrels $(n=2)$, Norfolk Terriers $(n=1)$, Miniature Dachshunds $(n=1)$, and Maltese $(n=2)$, and their ages were $9.3 \pm 6.5$ months; the bodyweight was $3.3 \pm 0.8 \mathrm{~kg}$. The concentration of VIP in the saphenous vein was $17.75 \pm 13.88 \mathrm{pg} / \mathrm{ml}$; on the contrary, the concentration of VIP in the portal vein was $29.7 \pm 20.29$ $\mathrm{pg} / \mathrm{ml}$. There was no significant difference in the VIP concentration between veins.

Conclusion: There was no difference in the VIP concentration between the portal and saphenous veins, suggesting a
\end{abstract} non-association between VIP and the PSS, in the absence of portal hypertension.

Keywords: Dogs, Portosystemic shunt, Vasoactive intestinal peptide.

\section{Introduction}

In mammals, the portal venous system collects blood from the major abdominal organs and delivers nutrients, bacteria, and toxins from the intestines to the liver to be further metabolized or excreted. Additionally, the portal blood carries approximately one-half to two-thirds of the oxygen supply to the liver, along with specific hepatotrophic factors (McMichael, 1937; Vollmar and Menger, 2009). The congenital portosystemic shunt (PSS) is a common hepatic vascular anomaly in dogs. Previous studies have shown a relationship between systemic inflammation and hepatic encephalopathy, which is the most significant factor for morbidity and mortality. Interleukin, proinflammatory cytokines, and portal lipopolysaccharide with Toll-like receptor-4 mediated cytokine activations are significantly elevated in dogs with PSS compared to healthy dogs (Kilpatrick et al., 2014; Tivers et al., 2015b); the attenuation of congenital PSS reduces these markers of inflammation. The Tivers study was only based on markers and blood parameters, and not on actual cellular evidence of inflammation (Tivers et al., 2015a). Vasoactive intestinal peptide (VIP) is a peptide hormone, produced by many tissues including the gut and pancreas, that stimulates contractility of the heart, causes portal vasodilation, and decreases arterial blood pressure (Hellstrand et al., 1985). This peptide was also considered to be a neurotransmitter, and it has been reported that an increase in blood VIP concentration is simply the result of an overflow of excessive secretion from nerve endings (Corcoran et al., 1999). In cats, elevated levels of VIP were measured in portal venous effluent following either direct or reflex activation of the vagal innervation of the stomach, mechanical stimulation of the small bowel mucosa, stimulation of the pelvic nerves to the colon, and mechanical stimulation of the rectal mucosa (Fahrenkrug et al., 1978). Additionally, the corelease of VIP and peptide histidine isoleucine into the portal vein has been demonstrated in dogs following vagal nerve stimulation; more importantly, increased portal VIP may cause a reduction in portal inflow resistance (Yasui et al., 1987). Collectively, VIP may be involved in the modulation of portal vein pressures. Thus, the VIP concentration may be higher in the portal vein compared to a peripheral vein in canine PSS. This pilot study aimed to evaluate the concentrations of VIP in the portal and peripheral veins in clinical settings of canine PSS in the absence of portal hypertension.

\footnotetext{
*Corresponding Author: Mitsuhiro Isaka. School of Veterinary Medicine, Rakuno Gakuen University, 582 Bunkyodai-Midorimachi, Ebetsu, Hokkaido 069-8501, Japan. 


\section{Materials and Methods}

Six client-owned dogs (three male and three female including one spayed female) were included in this study. All dogs were previously diagnosed with an extrahepatic portosplenic shunt. Premedication included intravenous fentanyl citrate $(5 \mu \mathrm{g} / \mathrm{kg})$ and intramuscular atropine $(0.05 \mathrm{mg} / \mathrm{kg})$. The dogs were anesthetized intravenously with propofol $(6 \mathrm{mg} / \mathrm{kg})$ and endotracheally intubated. Under general anesthesia maintained with sevoflurane, the measurement of portal pressure and collection of blood samples were carried out by cannulation of the anterior intestinal vein. Blood samples $(2 \mathrm{ml})$ were taken from the saphenous and portal veins during PSS ligation surgery with an ameroid constrictor, to evaluate and compare the VIP concentration in both samples. The collected sera were stored at $-80^{\circ} \mathrm{C}$ until measurement. VIP was measured using a commercial canine enzymelinked immunosorbent assay kit (MyBioSource, San Diego, CA) with a sensitivity of $1.0 \mathrm{pg} / \mathrm{ml}$. Statistical difference between two groups was carried out by unpaired Student's $t$-test, using Statcel 4 software (OMS Publishing, Saitama, Japan). Statistical significance was set at $p<0.05$.

A parametric, two-group comparison was carried out by paired Student's $t$-test and was considered significant when $p$-value was $<0.05$.

Ethical approval

All procedures were approved by the Rakuno Gakuen University, School of Veterinary Medicine Institutional Animal Care and Use Committee (approval No. VH20B9).

\section{Results and Discussion}

In our cohort, the breeds included Mongrels $(n=2)$, Norfolk terriers $(n=1)$, Miniature Dachshunds $(n$ $=1)$, and Maltese $(n=2)$, and their ages were $9.3 \pm$ 6.5 months; the body weight was $3.3 \pm 0.8 \mathrm{~kg}$. The portal pressures pre- and post-ligation were $8.75 \pm$ $3.3 \mathrm{mmHg}$ and $14.25 \pm 2.87 \mathrm{mmHg}$, respectively. There was no significant difference between the preand post-pressure. The venous VIP concentration was $17.75 \pm 13.88 \mathrm{pg} / \mathrm{ml}$, and the portal VIP concentration was $29.7 \pm 20.29 \mathrm{pg} / \mathrm{ml}$ (Fig. 1). There was no marked discrepancy in VIP concentrations between the portal and saphenous veins.

Our experimental data showed no statistical difference in the concentration of VIP between peripheral and portal veins in dogs with PSS that did not suffer from portal hypertension; this was supported by the findings of a previous study in rats (Johnson et al., 1994). In that study, mesenteric congestion alone played a minor role in the pathogenesis of portal hypertension, which may instead be related to the PSS of vasoactive substances other than glucagon and VIP in rats (Johnson et al., 1994). On the contrary, VIP could decrease the portal pressure in rats, with portal hypertension caused by high-frequency electrical vagal nerve stimulation

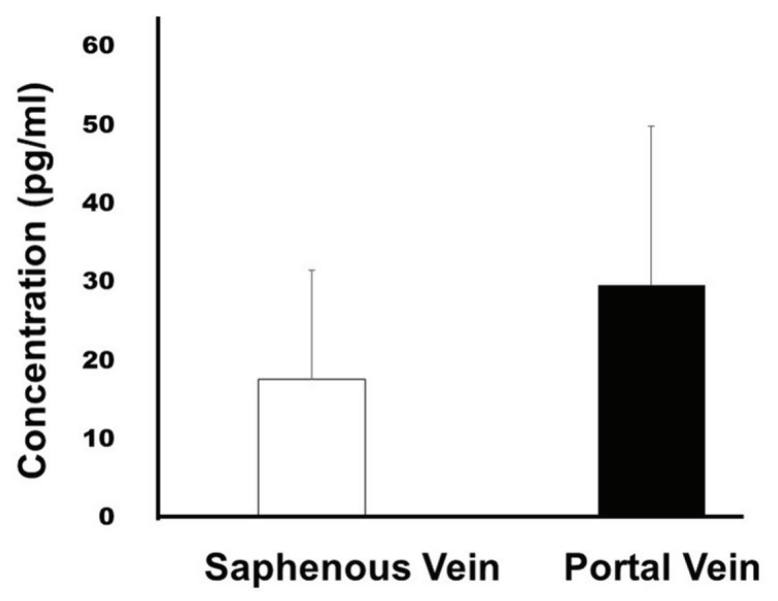

Fig. 1. The concentration of VIP between the portal and saphenous veins.

(Bockx et al., 2012). In our study, we did not confirm nerve function, and clinical evaluation did not show portal hypertension. VIP may not be associated with portal pressure in canine PSS in the absence of portal hypertension. However, dogs with PSS rarely have portal hypertension prior to surgery but can develop it postoperatively. Thus, we thought to follow-up the patients from this cohort to measure the peripherally VIP concentrations in the future. Our study has certain limitations; there were no dogs with portal hypertension, the sample size was small, and there were no controls. Larger prospective clinical trials are required for evaluating and comparing VIP concentrations between dogs with and without portal hypertension.

\section{Conflict of interest}

The authors declare that there is no conflict of interest. Authors'contributions

Dr. Mitsuhiro Isaka performed the surgery, conceived the study design, and prepared the manuscript. Dr. Hiroshi Ueno performed the surgery with Dr. Isaka and approved the final version.

\section{References}

Bockx, I., Verdrengh, K., Vander Elst, I., van Pelt, J., Nevens, F., Laleman, W. and Cassiman, D. 2012. High-frequency vagus nerve stimulation improves portal hypertension in cirrhotic rats. Gut. 61, 604612.

Corcoran, B.M., Jarvis, S., Hahn, C.N. and Mayhew, I.G. 1999. The distribution of nerve fibers immunoreactive for vasoactive intestinal peptide, calcitonin gene-related peptide, substance $\mathrm{P}$ and dopamine $\beta$-hydroxylase in the normal equine larynx. Res. Vet. Sci. 67, 251-259.

Fahrenkrug, J., Haglund, U., Jodal, M., Lundgren, O., Olbe, L. and de Muckadell, O.B. 1978. Nervous release of vasoactive intestinal polypeptide in the gastrointestinal tract of cats: possible physiological implications. J. Physiol. 284, 291-305. 
Hellstrand, P., Fahrenkrug, J., Uddman, R. and Järhult, J. 1985. Role of vasoactive intestinal polypeptide (VIP) in the neurogenic vasodilatation of the portal vein in the rabbit. Regul. Pept. 12, 309-316.

Johnson, T.J., Quigley, E.M., Shaffer, C.J., Adrian, T.E. and Rikkers, L.F. 1994. Effects of portal vein stenosis and superior mesenteric vein ligation on mesenteric venous pressure and porta-systemic shunting in the rat. J. Invest. Surg. 7, 477-483.

Kilpatrick, S., Gow, A.G., Foale, R.D., Tappin, S.W., Carruthers, H., Reed, N., Yool, D.A., Woods, S., Marques, A.I., Jalan, R. and Mellanby, R.J. 2014. Plasma cytokine concentrations in dogs with a congenital portosystemic shunt. Vet. J. 200, 197199.

McMichael, J. 1937. The oxygen supply of the liver. Exp. Physiol.127, 73-87.
Tivers, M.S., Handel, I., Gow, A.G., Lipscomb, V.J., Jalan, R. and Mellanby, R.J. 2015a. Attenuation of congenital portosystemic shunt reduces inflammation in dogs. PLoS One. 10, e0117557.

Tivers, M.S., Lipscomb, V.J., Smith, K.C., Wheeler-Jones, C.P. and House, A.K. 2015 b. Lipopolysaccharide and toll-like receptor 4 in dogs with congenital portosystemic shunts. Vet. J. 206, 404-413.

Vollmar, B. and Menger, M.D. 2009. The hepatic microcirculation: mechanistic contributions and therapeutic target in liver injury and repair. Physiol. Rev. 89, 1279-1339.

Yasui, A., Naruse, S., Yanaihara, C., Ozaki, T., Hoshino, M., Mochizuki, T., Daniel, E.E. and Yanaihara, N. 1987. Corelease of PHI and VIP by vagal stimulation in the dog. Am. J. Physiol. 253, 13-19. 